\title{
Vida familiar y trayectorias académicas: una aproximación biográfica en una universidad chilena
}

\author{
Francisca Ortiz Ruiz \\ Centro de Investigaciones Socioculturales,Universidad Alberto Hurtado, \\ Santiago, Chile. \\ Email: franortizruiz@gmail.com
}

\begin{abstract}
Resumen $^{1}$ : El objetivo de la presente investigación consistió en analizar las trayectorias académicas relacionadas con la vida familiar de hombres y mujeres que trabajan en las ciencias exactas. Se trabaja con un enfoque metodológico de carácter biográfico, a través de la construcción de las historias de vida de 17 académicos/as. Se encuentra evidencia de que la trayectoria académica está totalmente imbricada con la vida familiar, de pareja y personal de cada sujeto. Se concluye que las desigualdades de género entre mujeres y hombres también se expresan en diferentes momentos de la vida en una carrera académica. vida, ciencia.

Palabras claves: Trayectoria académica, vida familiar, academia, relatos de
\end{abstract}

\section{Family life and academic trajectories: a biographical approach in a Chilean university}

\begin{abstract}
The objective of the present investigation was to analyze the academic trajectories related to the family life of men and women who work in the exact sciences field. We work with a methodological approach of a biographical character, through the construction of life histories of 17 academics. There is evidence that the academic trajectory is deeply imbricated within the family, couple and personal life of each subject. It is concluded that gender inequalities between women and men are also expressed at different points in life in an academic career.
\end{abstract}

Keywords: Academic trajectory, family life, academy, life stories, science.

\section{Vida familiar e percursos académicos: a abordagem biográfica emumauniversidade chilena}

Resumo: O objetivo deste estudo foi analisar as trajetórias acadêmicas relaciona das à vida familiar de homens e mulheres que trabalham nas ciências exatas. Ele trabalha com uma biográfico abordagem metodológica a través da construção de histórias de vida de 17 acadêmicos / as. Evidências de que formação acadêmica está completamente entrelaçadacom a vida familiar, casal e pessoal de cada sujeito. Conclui-se que as desigualdades de gênero entre homens e mulheres também são expressos em diferentes momentos da vida em uma carreira acadêmica. 
Polis, Revista Latinoamericana, $N^{\circ} 47,2017$

Palavras-chave: formação acadêmica, a vida familiar, universidades, histórias de vida, ciência.

\section{Introducción}

La academia universitaria es el lugar por excelencia en el cual se construye ciencia en Chile (Gilbert, 2011). Es un contexto en el cual las trayectorias de los/as académicos/as se desarrollan, generando mayores niveles de productividad científica a nivel nacional e internacional. Allí la presencia de mujeres es menor que en otros contextos, sobre todo si revisamos ámbitos más ligados a las ciencias exactas (química, física, astronomía) o matemáticas (ingeniería civil), donde ocupan cerca de un tercio de los puestos disponibles (CONICYT, 2013). El principal antecedente que promueve la mantención de esta desigualdad, es la asociación del rol de la mujer al ámbito doméstico y la familia (PNUD, 2010). Así las trayectorias académicas y la vida familiar, son ámbitos que parecieran estar entrecruzados en las historias de vidas de los/as académicos/as que trabajan en las universidades.

Esta investigación se planteó el objetivo de analizar las trayectorias académicas en relación con la vida familiar de los hombres y las mujeres que trabajan en las ciencias exactas, desde una perspectiva biográfica del fenómeno. Para ello, se realizó una revisión bibliográfica sobre las formas en que se han estudiado la relación entre la vida familiar y las trayectorias académicas. Así se problematizan principalmente dos líneas de investigación: (1) interaccionismo simbólico y (2) capital cultural y habitus. Ambas líneas consideran la vida familiar solo a nivel secundario en sus textos, por lo que el dialogo que se propone entre estas, en este artículo es tan relevante teóricamente. Aquí se propone que la vida familiar debiera ser prioridad aser considerada en la misma definición de lo que es la trayectoria académica ${ }^{2}$; dado que se podría decir que la vida familiar forma parte constructiva de la contextualidad de la fabricación del conocimiento científico (Knor Cetina, 2005).

Por otro lado, esta investigación considera la perspectiva de géne$\mathrm{ro}^{3}$ debido a que en la revisión bibliográfica se evidenciaque en este tema existen estudios sobre todo centrados en los discursos de las mujeres, sin considerar a los hombres. A lo que se suma la necesidad recurrente de realizar investigaciones de las trayectorias académicas longitudinales, o que incorporen la noción de temporalidad de alguna u otra forma (Selva et. al 2011, p.236; Longo, 2009, p.139). De lo cual se busca hacer cargo por medio de la recolección de los relatos de vida de 17 casos de académicos/as de una facultad de química de una universidad chilena.

Se concluye que existen instancias de tensiones (que involucran prácticas y estrategias), pero también existen momentos de sinergia y re- 
troalimentación entre estas dos áreas, que muchas veces se presentan analíticamente como dimensiones distintas, siendo que en realidad se encuentran completamente entrelazadas por la cotidianidad. Los tres ámbitos son espacios en los que se visibilizan estas tensiones, sinergias y retro-alimentaciones, pero no es lo único, ya que surge la relevancia de considerar en un futuro no solo la vida familiar, sino que también la vida personal (el hecho de tener espacios para ellos/as mismos/as, pasatiempos, deportes, entre otros) y la vida de pareja ${ }^{4}$.

\section{Sobre la vida familiar en la academia}

El estudio sobre los/as académicos/as en la producción del conocimiento, se centra principalmente (si es que no únicamente) en la universidad (Lavados, 2006), debido a que es aquí donde se lleva a cabo la construcción de las identidades de los/as científicos/as. Aun así, las investigaciones que indagan sobre las experiencias personales y la vida cotidiana de quienes se dedican a la ciencia y/o trabajo académico, es casi inexistente (Martínez, 2012, p.140; Berríos, 2005, p.360), área en la que esta publicación busca ser un aporte. En tal ambiente el ideal predominante es que las personas le "dan prioridad total al trabajo y no tengan intereses o responsabilidades fuera” (Fox, 2010, p.102) de su profesión, lo que muchas veces puede tensionarse con la vida familiar.

En Chile hay algunas investigadoras que han problematizado la existencia de estas diferencias según género, en el comienzo de una carrera científica (Bernasconi, 2010), en el desarrollo de las trayectorias laborales académicas (Mora, 2010; Sonnert y Holton, 1996), y en el acceso y la participación a la comunidad científica (Stefoni, 2010). Tal como dirá Claudio Ramos "el campo presenta claros clivajes marcados por el género" (2012,p.38), al estudiar el comportamiento de las ciencias sociales en Chile. Así, en la academia es latente la posibilidad de que las desigualdades de género se trasladen no solo al ámbito científico, sino que también se manifiesten desde equilibrios o tensiones en el desarrollo de sus vidas familiares.

Karin Knorr Cetina (2005), a partir de su inserción en variados laboratorios escribe que "las selecciones que operan al interior de un laboratorio, responden a una contextualidad más allá del sitio concreto de la producción de tal conocimiento" (p.176). Este último es construido en "campos transcientíficos variables", que son "atravesados y sostenidos por relaciones de recursos, y no los grupos de pertenencia profesional como las "comunidades científicas”, constituyen las redes de las relaciones sociales en las cuales los científicos sitúan su acción de laboratorio” (Knorr, 2005,p.321). La práctica científica que se da en un laboratorio, tiene de forma inherente un carácter de indeterminación y contingencia contextual, siendo así una construcción social (Kreimer, 1999,p.167) conllevando considerar la contextualidad del trabajo científico, que en esta investigación será específicamente la vida familiar. 
En el área de la investigación cuantitativa, el estudio de la relación entre los/as académicos/as y sus vidas familiares ha ido en aumento, sobre todo en indagaciones sobre las barreras y los elementos que favorecen el equilibrio entre ambos (Chinchilla et. al, 2006,p.1; Cerros y Ramos, 2011,p.231232; Rodigou et. al, 2010). Por ejemplo en Chile, Martínez evidencia que la "tensión entre lo laboral y lo familiar "tiene que ver sobre todo con los roles y los tiempos, [las cuales] muchas veces (...) son antagónicos entre sí” (Martínez, 2012,p.154). Existen entonces varios factores que pueden incidir en la conciliación entre las trayectorias académicas y las vidas familiares: los ciclos de vida (Martínez, 2012), la estructura familiar (Solé y Parella, 2004) y las dinámicas familiares (O’Laughlin y Bischoff, 2005; Valdés, 2007, 2009; Ortiz y Mejía, 2009).

Una de las aristas cada vez más estudiada, es la vivencia de la maternidad de las mujeres y su relación con el desarrollo de sus trayectorias académicas (Arendell,2000, p.1201). Existe evidencia que respalda un mejor éxito en el desarrollo de las trayectorias de las mujeres académicas, debido al rechazo o una posposición del matrimonio y la tenencia de hijos/as (Mason y Goulden, 2004, p.100; Kyvik, 1990, p.158); mientras que los efectos de las distintas formaciones familiares varían según el género (Mason y Goulden, 2004, p.100). Sobre este tema, aún quedan dudas, pues también hay evidencia de la inexistencia de una asociación significativa entre el matrimonio y la tenencia de hijos/as, y la carrera de mujeres científicas (Cole et. al, 1987,p.125). Según Palomar (2009), la maternidad que tensiona el mundo académico, aun así genera espacios de cooperación con ciertas redes para afrontar esa situación.

\section{El estudio de las trayectorias académicas}

Dada la gran cantidad de elementos a considerar al momento de estudiar la conciliaciónde la vida familiar con la academiaen el contexto universitario, es central la definición que se utiliza en esta investigación sobre el concepto de trayectoria académica. La noción de trayectorias académicas es abordada desde dos perspectivas teóricas: en primer lugar, desde el interaccionismo simbólico, y en segundo lugar, desde el capital cultural y el habitus.

\section{Perspectiva desde el interaccionismo simbólico}

En 1937 Everett Hughes investigo cómo se producen los cambios en un mismo trabajo o hacia otros trabajos de mayor prestigio (Hughes, 1937,p.409), lo que luego termina siendo uno de los estudios más citados en torno al ámbito laboral de las personas (Heath, 1984 p.218). El autor indagaen las contradicciones que existen al momento de definir el estatus en una ocupación, concluyendo que una “carrera” es:

“objetivamente, una serie de jerarquías y cargos claramente definidos... las consecuencias típicas de la posición, los logros, las res- 
ponsabilidades, e incluso las aventuras (...). Subjetivamente, una carrera es la perspectiva móvil desde la cual el individuo ve su propia vida como un todo e interpreta el significado de sus diversos atributos, acciones, y aquello que le sucede” (Hughes, 1937, p.413)

En 1948, Oswald Hall por su parte, investiga la desagregación de los estados que componen los ajusten a través de la carrera en un largo periodo de tiempo. Ello sobre todo considerando las "redes de instituciones, organizaciones formales y relaciones informales, en las que se práctica esa profesión” (1948, p.327). Estudios que más tarde, serán retomados por Howard Becker desde la micro-sociología (2005; Becker y Strauss, 1956).

Howard Becker escribe el libro Outsiders (2005) desde la perspectiva de la sociología de la desviación, explorando las carreras de los consumidores de marihuana y los jazzistas de baile. Para él la carrera "se refiere a la secuencia de movimientos de un puesto de trabajo a otro que hace un individuo que se desplaza dentro del sistema ocupacional” (p.43), lo cual convive con cierta "contingencia ocupacional” (p.43) que envolvería hechos objetivos y más subjetivos, como los puntos de vista, motivaciones y deseos de la persona. En el caso particular que estudia, Becker (2005) identifica distintas etapas progresivas, controles a nivel social y subculturas, a través del rescate de la historia de los propios individuos.

El componente familiar suele surgir en sus investigaciones, en las experiencias de construcción de sus carreras (en ambos ámbitos), pero sobre todo al estudiar los jazzistas de música de baile:

"Finalmente, la familia del músico (ya sea la de nacimiento o la que forma al casarse) tiene un efecto decisivo en su carrera. Padres y esposas no son necesariamente músicos y, en tanto "marginales", suelen no comprender la naturaleza del vínculo entre el músico y su trabajo. Los malentendidos y desacuerdos que surgen en el seno del hogar muchas veces provocan cambios en el rumbo profesional de un individuo y, en algunos casos, son el punto final del recorrido.” (Becker, 2005, p. 124)

El matrimonio y la conformación de la familia impulsan el desarrollo de una carrera o son el generador de problemas entre lealtades e ideas de sí mismo que entran en conflicto (1955); según lo cual se determinaría la duración y rumbo que tome la carrera del jazzista. El autor plantea entonces la existencia de un alto nivel de involucramiento entre lo laboral y lo familiar (Becker y Strauss, 1956; Becker, 2005), pero no detalla precisamente esta relación.

\section{Perspectiva desde el planteamiento del capital cultural y el habitus}

Desde esta perspectiva Pierre Bourdieu, al escribir el libro "Homo Academicus" describe detalladamente las lógicas de poder y las estrategias al interior del campo académico (Bourdieu, 2012a), comprendiendo a 
este último, como un contexto en que las diferencias son parte del día a día, estrechamente ligadas al capital incorporado (habitus), con asociación a los orígenes sociales y geográficos, visualizado:

“A lo largo de una trayectoria académica particularmente exitosa (como lo atestigua la consagración por medio del concurso general) y de una carrera universitaria completa, y especialmente en ocasión de cada una de las elecciones entre secciones, entre opciones, entre instituciones (...) donde el espacio de los posibles se estrecha.” (Bourdieu, 2012a,p.76)

Así desde esta perspectiva se realza la edad, las diferencias entre las familias de origen y las tensiones existentes en el contexto académico, caracterizada por una competencia constante entre los/as académicos/as por el poder. El cual "no puede ser acumulado y mantenido, sino al precio de un gasto constante e importante de tiempo” (Bourdieu, 2012a,p.130). Así las trayectorias académicas se construyen de formas distintas, según las ventajas heredadas de cada uno, y sus ventajas merecidas (de negociación de beneficios materiales y simbólicos) (Bourdieu, 2007,p.150). Quienes trabajan en la academia son parte de aquella illusio que mantiene el juego por ser una mayor autoridad científica (Bourdieu, 2012b,p.23), es decir, ostentar un mayor nivel de capital acumulado que los demás y por ello están en una posición más consolidada a partir de su reputación en el medio, su capacidad de convocar, el valor distinto de su trabajo y la originalidad del mismo. En síntesis, para obtener un mejor posicionamiento en la academia, es necesaria una gran inversión de tiempo en esta área por sobre las otras actividades de la vida de las personas. Desde esta perspectiva, es importante la influencia de la familia de origen, siendo lo que genera adhesión a ciertos valores en un grupo y su cohesión. Pero no se indaga más sobre la familia actual de la persona (o de destino), aunque menciona que "como se puede ver en el celibato o el divorcio, por un lado, y en el tamaño de la familia [de origen], por el otro, que contribuyen bastante a producir la oposición principal del campo” (2012a,p.71). La lógica propia de la academia así es el prestigio intelectual y el éxito científico, por medio de la constante inversión de tiempo (Bourdieu, 2012a,p.130-136).

En síntesis, los distintos autores mencionados (de ambas perspectivas), consideran breve y secundariamente a las vidas familiares de los sujetos. Su relevancia para mencionarlo, radica en que en sus investigaciones evidencian situaciones de tensión entre las trayectorias académicas y la vida familiar. Pero siempre se mantiene la vida familiar en un segundo plano, ya que no era el tema central que buscaban responder, sino un tema que emergió en su momento.

\section{Metodología}

Para comprender como son las trayectorias académicas en relación con la vida familiar de los hombres y mujeres que trabajan en las ciencias 
exactas, se indagó desde una perspectiva cualitativa (Taylor y Bogdan, 2000) por medio del estudio de caso múltiple (Stake, 2013,p.161). Se decidió estudiar entre las ciencias exactas, una facultad de química, debido a que es una carrera en donde predomina la concepción de la ciencia fuertemente ligado al positivismo y el contraste de hipótesis (Holt 1970), sumado a altos niveles de competitividad según el prestigio (Morris, 2010; Smart y McLaughlin, 1985).

Se realizaron 3 encuentros en general con 17 casos, divididos entre académicos asistentes, asociados y titulares, además de considerarse en igual proporción hombres y mujeres. El trabajo de campo se realizó entre agosto del 2014 y enero del 2015, desde el enfoque biográfico (Ferraroti, 2011; Guerra y Skewes, 1999; Pujadas, 2002; Sautu, 2004). Así se reunieron los relatos de vida (Kornblit 2004; Cornejo, Mendoza y Rojas 2008) de todos los casos, buscando explorar la vida cotidiana y los episodios vividos, considerando las emocionalidades de las personas al responder (Bernasconi 2011; Sharim, 2005). Por último, el análisis se realiza a partir de la propuesta comprensiva de Bertaux (1981, 1989; Kornblit, 2004,p.22-23).

\section{Resultados}

Luego de la recolección de los datos y su análisis, se identificaron cinco momentos ${ }^{5}$ en el tiempodentro de la trayectoria académica en general: (1) comenzar a interesarse por las ciencias exactas, (2) el rito de iniciación: el Doctorado, (3) dentro de la universidad y de la academia: ser académico/a asistente, (4) lograr la estabilidad laboral, económica y familiar: ser académico/a asociado y (5) La consolidación en la academia: ser académico/a titular. En adelante se describen brevemente cada uno de estos, con énfasis en la interrelación entre la vida familiar y el ámbito laboral, además de la emergencia de la vida personal y de pareja.

\section{Comenzar a interesarse por las ciencias exactas}

Una de las primeras preguntas que se les hizo a todos/as los/as entrevistados/as, era por el origen del gusto por las ciencias exactas, y específicamente a lo que se dedicaban hoy en día: la química. Además de ser una buena entrada con ellos/as, en general se pueden evidenciar la presencia de personas, sensaciones y experiencias en sus relatos. Algo que todos/as destacaron fue que sus padres habían estudiado algún tipo de carrera universitaria, teniendo algún título profesional y varios mencionaban que era el contexto familiar ligado a la docencia o a la investigación, lo que provocaba que terminarán dedicándose a la ciencia. La profesora asociada Camila comenta aquello es el "espíritu

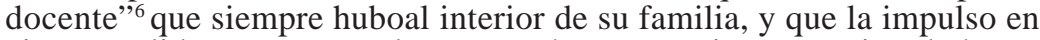
cierta medida a que naturalmente tenía que seguir ese camino de hacer clases trabajando en la academia (debido que es el único espacio en donde podía dedicarse a ello). 
En otros casos la importancia de la madre era alta, ya que era un agente clave al momento de esclarecer que es lo que harán a futuro. El académico Agustín comentaba que su madre fue la que "sabiamente” le recomendó que estudios seguir, por lo que hoy en día le agradece. La presencia de los padres en la decisión porque carrera universitaria seguir, no era mencionada; lo que se puede contrastar con el hecho de que ya desde el siglo XVII en Chile, la preocupación por la educación de los/as hijos/as y su crianza era la labor de las mujeres (Pereira 2007, p. 307; López y Findlin 2012, p. 150).

Ligando esta etapa, con un componente fuertemente emocional, además del lazo que tienen con las sensaciones que les producen personalmente las labores de docencia (el “espíritu docente”) y la investigación (“curiosidad”, “pasión”, el “deseo de comprender” y “materiales en los laboratorios”).

El inicio de la carrera académica los/as entrevistados/as comentaban que era doble (y en sus propias palabras): el "informal” y el "formal”. El primero era cuando la persona decidía de forma personal dedicarse a la docencia y/o investigación, para lo cual tenían que trabajar en la universidad, comenzando generalmente por la realización de ayudantías de cátedras y/o asistencias de investigación. En segundo lugar, el inició formal existe cuando hay un acuerdo contractual entre la universidad y el/la académico/a de forma remunerada durante un plazo indefinido o con proyección a futuro. Pero la única forma de lograr tener un inició formal en la trayectoria académica, es lograr pasar el requisito más importante: tener el Doctorado.

\section{El rito de iniciación: el Doctorado}

En la trayectoria académica de todos/as los/as entrevistados/as, había un antes y un después marcado por la realización del Doctorado es un periodo marcado para muchos por los problemas económicos, y la búsqueda de soluciones a través de la postergación de la tenencia de hijos/as, o en otros casosde la indagación deotros trabajos extras para aumentar el sueldo, (lo que dependía siempre del nivel de apoyo de parte de la pareja). Hombres y mujeres diferenciaban en sus respuestas, ellos describían el doctorado como una experiencia enriquecedora en materia de conocimiento, pero que aquello había traído consigo altos costos. En cambio, las mujeres al realizar sus doctorados hablan más bien desde la emocionalidad, lo que significó para ellas y la postergación de la maternidad, o en algunos casos en que quedaron embarazas en tal periodo, la complicación que significo lograr armonizar los tiempos entre la familia y sus tesis que "no podían parar por eso”.

Habían cinco razones por las cuales los/as académicos/as decidieron realizar el doctorado: (1) es un pre-requisito obligatorio para ingresar a la academia, (2) por ser parte del "centro de comodidad" de la gente continuar estudiando, (3) para poder desarrollar una investigación sobre su tema 
de interés y que le apasiona, (4) por tener la oportunidad de ser becados o enviados por la universidad, y luego compensados con años de trabajo asegurado dentro de la misma institución, e (5) incluso por la posibilidad de que al hacer el doctorado se podían financiar la vida cotidiana a través de las becas.

Las experiencias vividas en este periodo fueron muy distintas según tenían o no pareja, si tenían hijos/as pequeños/as, si los tuvieron durante el Doctorado o si los/as hijos/as nacieron después de retornar a Chile. Pareciera ser que la dificultad de lograr tales estudios aumentaba en la medida que se realizará el Doctorado en el extranjero (sin redes de apoyo familiares), más aun siendo una mujer embarazada (dado que tenía que continuar en cierta medida con sus estudios y su tesis que no se interrumpe) o estando con niños/as recién nacidos/as (que necesitan más tiempo en cuidados). Las mujeres se sentían con mucha culpa (como dice Ignacia "es muy difícil, muy difícil no tener culpa”) hasta hoy, por no haber podido dedicar tanto tiempo de calidad con su familia y específicamente con sus hijos/as, dado la constante presencia que requería la realización de sus estudios. La descripción de que fue "muy movido todo" o "estresante, yo corría para todas partes" se repite en varios relatos de mujeres, sobre todo dependiendo de la cantidad de tiempo que tenían que disponer para sus labores (desde amamantar o la guardería, hasta postular a unos fondos o escribir la tesis). Sobre este tema, uno de los académicos asociados comenta que en la academia "para llegar a los niveles altos, implica sacrificios fuertes" en lo personal, haciendo referencia a la dificultad que tenía para conciliar lo académico y el familiar. Dado que el sacrificio que implica lograr obtener el Doctorado,varios optan por su postergación, o la de tener hijos/as, o incluso eliminar la opción de ser padres de forma decidida.

\section{Dentro de la universidad y la academia: ser académico/a asistente}

Al finalizar el Doctorado y lograr incorporarse a una universidad, de tal forma de investigar o desempeñarse como profesores/as, vuelven a la institución académica. Volver es un momento complicado, sobre todo porque llegan con la sensación de tener que comenzar de cero a generar redes, investigaciones y espacios de docencia; lo que se intensifica en el caso de las personas extranjeras. En estas últimas personas, la decisión de vivir en Chile estaba marcada no siempre por la elección de la universidad (siendo reconocida y con prestigio internacional), sino que había situaciones en que la vida familiar era la predominaba, cambiando el curso de la trayectoria académica completamente. Tal es la historia de Matías, quien al recibir ofertas de distintas partes del mundo, se decide por vivir en Chile, ya que tenía a la madre de su pareja en uno de los países vecinos y con cierto grado de complicación de salud; lo que termino imperando por las otras ofertas, resolviendo que la posibilidad de estar con ella en menos de 24 horas era la prioridad.

Así la nacionalidad de origen de la persona aparece como un elemento distintivo, dificultando contar con las redes de apoyo familiares (en su 
mayoría mujeres) y académicas, por lo cual terminan contratando a una empleadadoméstica para ayudar con esas labores Esta situación generalmente estaba más presente (era más mencionada como una tensión) en las trayectorias de las mujeres académicas, para quienes todo aquello era un tema a considerar cada vez que querían viajar a un congreso, hacer una ponencia, escribir un artículo y asistir a reuniones. Para ellas, la dependencia conforme a los eventos familiares en relación con la vida familiar, estaba siempre presente, lo que incluso era constantemente mencionado en las entrevistas, sin si quiera haber llegado a las preguntas que pretendían indagar en aquello en la pauta. En este periodo la existencia de una guardería es esencial para los/as académicos/as con niños/as recién nacidos, y sobre todo para las personas con otro país de origen, sobre todo considerando que los cuidados necesarios en la primera infancia son tan intensos.

En cuanto a la realización de las primeras docencias e investigaciones, reconocen que la vuelta los trajo a un "ecosistema académico" altamente competitivo, demandante y que promueve ser autoexigente constantemente. Es un lugar de "ritmo acelerado" con falta de espacios de reflexión, ya que como dirá Camila "tú necesitas divagar para investigar”. Un ejemplo ilustrativo es el caso de Javiera, quien cuenta que fue contratada para trabajar en la universidad, se adjudicó un proyecto de investigación, pero también quedó embarazada. En sus propias palabras, “fue duro ese verano... pero me fue bien (...) estaba con prenatal y yo seguía escribiendo, no parai’”. De hecho concluye que la academia finalmente es un espacio en el que desde sus inicios y dependiendo en parte del desarrollo de la vida familiar, es un espacio que "tiene mucho costo personal” que no siempre se visibiliza.

Además de las redes de apoyo en la familia (madres, tías, primas, hermanas) para conciliar la academia con lo familiar y la contratación de una trabajadora doméstica, la cercanía geográfica entre la casa, el lugar de trabajo, los familiares y el lugar de estudio de los/as niños/as fue de gran ayuda. Esto les permitía ahorrar un tiempo considerable, que podían direccionar para dedicar a la familia, a lo laboral, a sus parejas o para ellos/as mismos/as. Esto último surgió reiteradas veces en las entrevistas, el hecho de considerar no solo los tiempo de la vida familiar y la trayectoria académica, sino que también el tiempo destinado a la pareja y el personal (como por ejemplo para hacer algún deporte o un curso o un taller), que eran cada vez más extrañados por los/as académicos/as según el periodo en el que se encontraban.

El tiempo dedicado a la docencia, la investigación, extensión y gestión, para muchos era casi imposible de delimitar, en sus palabras las respuestas eran "24 horas”, “siete días a la semana”, y es que "uno vive en función de eso”. Sobre todo en los casos de los/as investigadores/as que se desempeñaban en la investigación en laboratorios, ya que al trabajar con organismos vivos, sus tiempos, no son los mismos que la jornada laboral, por lo que a veces puede tocar tener que ir a "alimentarlos" en horarios y días no considerados en un principio (fines de semana o en la noche). Lo mismo sucede en los casos en que dedican varias horas del día a la docencia, ya que su preparación y las correcciones de trabajos generalmente son 
más que las horas de trabajo en la oficina (sobre todo si es que es la primera vez que se dicta el curso). Apartir de ello, es que todo lo que no alcanzan a terminar en la universidad, suelen ser las cosas que se llevan como "trabajo para la casa", lo cual para Pedro a pesar de que "trato de no trabajar en casa, esa es la verdad, pero eso no impide que trabaje intelectualmente”.

En este periodo las mujeres se ven más agobiadas por los roles que deben (y/o quieren) cumplir, entre ser profesionales, mujeres y madres. Javiera comenta que es muy distinto ser una académica soltera y ser una académica con hijo, ya que cuando “no teni’ hijo, te dedicai’ 100\% a la pega”. Mientras que como dirá Camila, cuando eres madre “yo soy madre y soy más tonta (...) porque tu mente no puede estar en una cosa como estaba antes, está en un cosa y que la tarea, que el sicólogo, la sicopedagoga, el uniforme, los platos, la comida...”. Pero cuando logran superar la etapa “más movida”, varias de ellas reconocen como una profunda sensación de orgullo propio, ya que lograron sobrellevar todos los roles que tenían que realizar a la vez. A pesar de que el tiempo que tienen que dedicar a la crianza va disminuyendo en la medida en que van creciendo, en síntesis algunas estrategias de conciliación de los tiempos son: la cercanía geográfica, la guardería, respetar los horarios de trabajo diferenciándolos con los de la familia (al menos en el ideal) y la utilización de las redes de apoyo (generalmente parientes mujeres).Estrategias que serán útiles y cada vez menos recurridas, en la medida en que van alcanzando cierta estabilidad laboral, económica y familiar.

\section{Lograr la estabilidad laboral, económica y familiar: ser académico/a asociado/a}

Alcanzar la estabilidad en los espacios de desarrollo de la persona, conlleva varias cosas, pero una de las más destacadas por las personas para definir ese momento, era la relación de pareja. La similitud entre las disciplinas de cada uno (ciencias exactas) o de tipo de trabajo al ser ambos académicos, empatizan con el otro, y se han podido repartir de mejor forma las tareas domésticas. A pesar de ello, aún permanecen las mujeres como principales gestores de las labores de cuidado de otros (padres, abuelos, nietos, hijos) y de las tareas domésticas, repercutiendo en sus trayectorias académicas.

Como dirá Pedro “es mucho más fácil tener una pareja que entiende mucho más lo que uno habla (...) que entiende más los esfuerzos que se tienen que hacer". Pero también existen casos, como es el de Javiera, quien comenta que todo "giraba en torno a la competitividad", siendo un periodo “súper difícil” para ella, razones por las que más tarde,terminaría la relación. Así la pareja conlleva consecuencias en la trayectoria académica y viceversa, lo que fomentaba una preocupación en el caso de varios, sobre la posibilidad real de tener tiempo a disposición para compartir en pareja,demanda que no siempre pueden cumplir, sobre todo en los casos que hay niños/as pequeños/as. Como dice Nicolás "la vida de pareja es complicada, porque hay pocos espacios para la vida de pareja, es más la vida familiar... y cues- 
ta”. En esta etapa, todos/as los entrevistados/as tenían hijos/as ya adolescentes y/o universitarios, que implicaban menos tiempo de cuidados, de atención y una menor dependencia con respecto a sus padres; quienes solventaban este periodo con un mayor tiempo para la pareja (como ya mencionamos) y para sus labores profesionales.

De tal forma de solucionar el problema de con quien dejar a los/as niños/as, muchos/as optan por la contratación de una trabajadora doméstica puertas adentro (sensación de pérdida de privacidad) o afuera (disminución del tiempo con la pareja).Este paso es fundamental en sus biografías, ya que marca una solución y una estrategia que les ayuda a poder alcanzar este momento en sus trayectorias. Sin tener el apoyo de alguien que se dedique al cuidado y/o a las labores domésticas, los/as académicos/as (sobre todo las mujeres) reconocían no haber podido llegar a ser nombrados asociados; cargo para el cual se necesita una gran acumulación de productos científico de diverso tipo.A pesar de ello, Camila comenta que hacer cierto "tipo de cosas, la ropa, la colación y eso es una rutina y es impagable", porque le gusta mucho poder hacerlo, ya que es una forma de cuidar a sus hijas. En las labores domésticas las generaciones más jóvenes develan, a diferencia de las mayores, una mayor implicación en aquellas labores; no obstante, eso siempre es descrito como una “colaboración” o un “apoyo” a lo que realiza la mujer.

A pesar de que los/as niños/as crecieran, el hecho de que fueran a la guardería y pasaran tanto tiempo personal compartiendo en tales dependencia, hacíaque ellos/as consideraban a la "universidad como parte de la familia” como es en el caso de Camila. Ella reconoce que sus hijas se acostumbraron a ese espacio y a considerarlo otra casa en la que vivían, y en la que querían estar: "la universidad es muy centro de mi casa en ese sentido (...) la universidad es parte de la casa (...) es parte de mi familia”. El hecho de tener que estar varias horas en el mismo lugar de trabajo, significa que se entablan una gran cantidad de relaciones de amistades en lo laboral y que muchas veces llegan a ser más personales; además de ser las redes de contacto de futuras investigaciones o que al menos promueven la reflexión. Así el nivel de implicación entre la institución “Universidad” y su vida familiar, reafirma lo entrelazadas que se encuentran la trayectoria académica de alguien con los distintos escenarios de su vida (familiar, personal y de pareja).

Los casos entrevistados develan en varios momentos esta imbricación, pero no es hasta esta etapa de camino a la consolidación, que ello/as al ver sus trayectorias hacia atrás, resaltan esta pertenencia de forma más imbricada entre sus vidas familiares, personales y de pareja con sus propias vidas profesionales.

\section{La consolidación en la academia: ser académico/a titular}

Luego de ya tener una estabilidad en términos laborales, económicos y familiares, además de pasar por procesos de recaudación de antece- 
dentes (investigaciones, artículos ISI, ponencias, estancias, etc.), el siguiente paso luego de llegar a ser académico/a asociado/a es alcanzar el último nivel de la consolidación en una trayectoria académica: ser académico/a titular.

Este es el último momento en este camino, en la que todos/as desean/aspiran llegar.Según las entrevistadas la baja presencia de mujeres titulares (solo un tercio), se debía principalmente a que muchas deellas tenían la necesidad de dejar la universidad buscando dedicarle más tiempo a la vida familiar por sobre lo laboral. Ellas y ellos suelen destacar que hoy en día existe un aumento considerable en la incorporación de mujeres estudiantes y profesoras. Llama la atención, que a pesar de aquello, existen ciertas desigualdades de género a la base, que no están siendo consideradas. Cuando se hablaba de este tema con los entrevistados, ellos decían como Francisco (profesor titular) que es muy agradable trabajar con mujeres, debido a que ellas son más “capaces (...) más honestas”. Por otro lado, Javiera también comenta ellas son más “responsables”, develando como la incorporación de las mujeres ha sido un avance, pero que ha sido acompañado por una esencializaciónde los atributos femeninos en las relaciones laborales. Ellos sobre todo consideran que las ventajas de este ingreso, son ganancias debido a cualidades más ligadas a lo emocional en las relaciones, y no tanto a atributos profesionales.

Por otro lado, al entrevistar a los/as académicos/as titulares destacan en sus relatos tres ideas: (1) La constante comparación con sus primeros años y de cómo ha llegado a donde están actualmente, con un sentimiento de añoranza por los tiempos pasados a la vez de cierto orgullo por sus logros. Relevan el hecho de que ahora hay una mayor estructuración, en las exigencias (en publicaciones, investigaciones y cursos realizados), siendo que en palabras de Nicolás "la calidad humana años atrás era distinta. Ahora la competencia ha matado, digamos, la convivencia en todas partes”. (2) En segundo lugar, la producción masiva de artículos en inglés y estándar ISI que han tenido que ir acumulando, y que aumenta con los años. Tendencia que cada vez es de menor preocupación, ya que ahora la creación de artículos es más bien “por gusto” y no solo de forma acumulativa, sobre todo porque ahora el crecimiento e independencia de sus hijos (en los casos en que eran padres y madres) había ayudado a la producción académica. (3) El tipo de enseñanza que mencionan realizar, es muy distinto a como se realizaba anteriormente. Relevan que los años de trabajo en sus laboratorios, les han entregado un valor, en tanto son ellos quienes más conocen datos que aparecen en los libros, y lo cual es un gran orgullo y logro.

Además de estas tres ideas, el hecho de ser nombrado “Titular” es considerado un honor, un gran orgullo, es el momento en el que logran equilibrar sus investigaciones y docencia; logrando con ello una posición dentro del campo, que ya no es necesario competir. Ya no quedan más escalones por continuar, debido aque cuando uno es nombrado con tal categoría, "uno es profesor titular por toda la vida” (Javier). Es así la tranquilidad emocional de transcender por medio del desarrollo de un trabajo de 
toda una vida, y por el cual muchas tuvieron que sacrificar tiempo que podrían haber dedicado a la familia y/o a la pareja y/o a ellos/as mismos/as.

\section{Conclusiones}

Se concluye retomando el marco teórico que en cuanto al campo de la ciencia la existencia de elementos estructurados de este, tales como las publicaciones, las investigaciones, docencias; y elementos simbólicos, la ilusión de trascender a través de un artículo y ser alguien que aporta al conocimiento. Se evidencia también que las actividades administrativas y las labores relacionadas con la vida familiar son elementos que frenan el desarrollo de la ciencia, al igual que la perdida de espacios de reflexión, dadas las constantes exigencias de aumentar la producción en serie, siendo además cada investigador/a el/la encargado/o de su difusión y marketing.

Específicamente si volvemos a los planteamientos de Howard Becker (1955, 1956, 2005), quien al mencionar la contingencia en el sistema ocupacional como una noción secundaria a sus análisis, aquí evidenciamos la relevancia que tiene al momento de abordarde la relación entre las trayectorias académicas y la vida familiar. Asíla consideración de instancias de cooperación al interior del espacio académico, a través de ejemplos vistos como la contratación de trabajadoras domésticas, las guarderías, y las redes de apoyo familiar y laboral. Develando con ello que la comprensión de las trayectorias académicas, sin considerar la vida familiar del sujeto, no está completa y carece de comprensión, en tanto aquello forma parte central de la contextualidad del conocimiento científico (radicando en ello la novedad de esta investigación).

A la luz de los resultados, otro aporte generado es que visualizando las entrevistas desde la perspectiva teórica de Pierre Bourdieu (2007, 2012a, 2012b),destaca la comprobación de la existencia de varios aspectos estructurales del campo de la ciencia; mientras que desde el marco teórico del interaccionismo simbólico, se corroboro la existencia de instancias cooperativas entre los agentes. Pero la mayor novedad del estudio, radica principalmente en la propuesta de complementación de ambos enfoques en un contexto académico, en el que ambas visiones aportan al conocimiento de las trayectorias académicas. Por ejemplo, si mientras desde Bourdieu vemos la existencia de lógicas tensionadas en la vivencia de la parentalidad; desde Becker, se soluciona aquel problema, por medio de la sinergia y retroalimentación de otros vínculos familiares, como lo son la mamá, tía, prima, hermana, por mencionar algunos.

Así, podemos concluir a partir de los hallazgos, una propuesta más correcta de definición de las “trayectorias académicas" a la luz de estos resultados: son las narraciones (secuencias de eventos, experiencias y significaciones, entre otros) relacionadas con sus vidas familiares, personales y de pareja (cruzados por el género y la nacionalidad); desarrolladas en un espacio llamado la academia (inserto generalmente en la institución univer- 
sitaria), en que se juegan estrategias de competición y de cooperación. Así se releva que las trayectorias académicas no solo son lo que concierne al contexto universitario o institucional (como se evidencio en el marco teórico de esta investigación), sino que también es necesaria la consideración de la vida familiar, personal y de pareja, en tanto producen y coproducen variadas tensiones, sinergias y retroalimentaciones entre todos estos cuatro ámbitos.

Queda pendiente la posibilidad de hacer indagaciones de las categorías que surgieron de esta investigación, desde un análisis cuantitativo a mayor escala, logrando comparabilidad entre tipos de universidades, disciplinas y otros países. También la posibilidad de realizar observaciones sobrela competitividad entre los/as científicos/as y como se pueden tomar medidas para contrarrestar su impacto en las otras áreas de la vida de la persona. El estudio desde la perspectiva de género de las interacciones y las prácticas cotidianas en el laboratorio, relevando la importancia de las tecnologías y sus usos.Así la contribución de la presente investigación es en el debate que se da sobre la igualdad de género en el contexto de la ciencia de Chile, aportando la propuesta de la incorporación de una mirada interrelacionada a la noción de trayectorias académicas en conjunto con la vida familiar, personal y de pareja. 


\section{Notas}

${ }^{1}$ Los resultados de la investigación aquí presentada, son provenientes de la tesis (aprobada) de grado Magister en Sociología, de la P. Universidad Católica de Chile, y guiada por la Dra. Claudia Giacoman de la misma institución, a quien agradezco su apoyo y comentarios. Este proyecto fue financiado por el Fondecyt Iniciación n¹121245 "Las familias en Chile: El trabajo de parentesco y la generación de constelaciones familiares” a cargo de Herminia Gonzálvez (Investigadora Responsable) en el Centro de Investigaciones Socioculturales (CISOC), quien constantemente aporto activamente a lo largo del estudio. Por otro lado también contó con el patrocinio del proyecto FONDAP 15130009 "Centro de Estudios de Conflicto y Cohesión Social (COES)".

${ }^{2}$ Las dimensiones a estudiar de las “Trayectorias académicas” serán el posicionamiento dentro del campo (estrategias, cargos, tipos de contratos), los logros y las responsabilidades.

${ }^{3}$ En relación a la categoría "género" (Ortiz y Larrazabal, 2015; Ortiz y Gonzálvez, 2016) esta investigación se adscribe a la siguiente definición: "la construcción social basada en creencias, expectativas, valores, actitudes, comportamientos, roles y lo que se espera que sean y parezcan hombres y mujeres en una sociedad y en un tiempo determinado. El género no es estático ni determinado al nacer, sino que es dinámico a lo largo de la vida y es relacional. Por lo tanto, género es una categoría sociológica, así como otras como clase social, raza/etnia, etc.” (Simbürger y Undurraga, 2013, p.171). Mientras por "perspectiva de género", se comprenderá "construcción teórica que sirve de entorno de referencia a algunas de las más importantes problemáticas que atañen a las sociedad, familias y personas” (Gonzálvez, 2010, p.61). Así siguiendo la misma línea, en esta investigación se devela en la incorporación en la muestra de tanto hombre, como mujeres.

${ }^{4}$ Estas tres distinciones entre la "vida familiar", la "vida en pareja" y la "vida personal" son formas de nombrar tres momentos distintos en la cotidianidad que los/as entrevistados/as reconocieron tener. Ellos/as mismos/as los nombraban con esas palabras, de tal modo de diferenciarlos. La "vida familiar" radica en el tiempo que se pasa con familiares, y a quienes el/la entrevistado/a considere como parte de su familia, involucrando vecinos, parientes lejanos, sobrinos, entre otros. La "vida en pareja” es el tiempo que se pasa exclusivamente con la pareja y nadie más, como por ejemplo, salidas a comer o ir al cine, eran las más nombradas. Mientras que la "vida personal" sería el tiempo que se dedicarían para actividades y/o instancias que no involucraran a nadie más, que a ellos mismos. En este último, se solía recurrir a los ejemplos de ir al gimnasio o salir a jugar un partido de futbol o salir con amigos/as.

${ }^{5}$ Para el análisis de las tensiones y cooperación de la vida académica en relación con la vida familiar se realizó la construcción del relato de vida de todos/as los/as entrevistados/as hasta la entrevista. Se consideró así toda su biografía, por lo que algunos breves hallazgos recopilados no se relacionan directamente con uno de los cincomomentos en el que se encuentra, esto se debe principalmente a la diversidad y la riqueza de las historias de vidas (fueron organizadas para lograr una mayor facilidad de la lectura y una mejor comprensión de lo temporal en las trayectorias) 
${ }^{6}$ Desde aquí en adelante se realiza una gran cantidad de menciones de frases o palabras entre comillas, que son mantenidas tal cual como fueron dichas en las entrevistas por los/as académicos/as. No se detalla en todas quienes las dicen, ya que muchas son repetidas por varias personas. También se quisiera recalcar que todos los nombres son seudónimos. 
Polis, Revista Latinoamericana, $N^{\circ}$ 47, 2017

\section{Referencias bibliográficas}

Arendell, T. (2000). Conceiving and investigating motherhood: The Decade's Scholarship. Journal of Marriage and Family,(62), 1192-1207.doi: 10.1111/j.1741-3737.2000.01192.x

Becker, H. (1955). Interpreting Family Life in Context. EnH. Becker y R. Hill (eds.)Family, Marriage and Parenthood(pp. 1-49). EEUU: D.C Health and Company.

Becker, H. y Strauss, A. (1956). Careers, Personality, and Adult Socialization. American Journal of Sociology, (62), 253-263.doi: 10.1086/222002

Becker, H. (2005).Outsiders. Hacia una sociología de la desviación. Buenos Aires, Argentina: Siglo Veintiuno.

Bernasconi, O. (2010). Conocimiento científico y género: la "instalación” de las recién llegadas. Seminario Género y ciencia, Universidad Alberto Hurtado, Santiago, Chile.

Bernasconi, O. (2011). Aproximación narrativa al estudio de fenómenos sociales: principales líneas de desarrollo. Acta Sociológica, (56), 936. Recuperado de: http://www.revistas.unam.mx/index.php/ras/ article/view/28611

Berríos, P. (2005). El sistema de prestigio en las universidades y el rol que ocupan las mujeres en el mundo académico. Calidad en la educación,(23), 349-361.

Bertaux, D. (1981). Biography and Society. The life history approach in social sciences. Beverly Hills: Sage.

Bertaux, D. (1989). Los relatos de vida en el análisis social. En J. Aceves (comp.),Historia Oral. Parte II: Los conceptos, los métodos, (pp. 136-148). México:Instituto mora-UAM.

Bourdieu, P. (2007). Intelectuales, política y poder. Argentina: Ediciones Eudeba.

Bourdieu, P. (2012a). Homo Academicus. Argentina: Siglo Veintiuno.

Bourdieu, P. (2012b). Los usos sociales de la ciencia. Argentina: Nueva Visión.

Cerros, E. y Ramos, ME. (2011). El papel de la pareja en el desarrollo profesional. Revista Pequén (1), 83-92. Recuperado de: https:// $\mathrm{w}$ w w r e s e a r c h g a t e. n e t/p u b l i c a t i o n/ 245024717_EL_PAPEL_DE_LA_PAREJA_EN_EL_DESARROLLO _PROFESIONAL_DE_LAS_CIENTIFICAS_UNIVERSITARIAS 
Chinchilla, N., León, C., Torres, E. y Canela, MA. (2006). Frenos e impulsores en la trayectoria profesional de las mujeres directivas. En N. Chinchilla, C. León, E. Torres, y MA. Canela, (coord.)Documento de investigación $n^{\circ} 632$ (sin pp.). España: Universidad de Navarra.

CONICYT. (2013). Informe Encuesta Investigadoras Fondecyt. Beneficios de género Conicyt. En Conicyt (coord.) Documento de trabajo.Santiago, Chile: Asesorías Profesionales Etnográfica Ltda.

Cole, J. y H. Zuckerman. (1987). Marriage, motherhood and research performance in science. Scientific American,(256), 119-125. Recuperado de: https://www.ncbi.nlm.nih.gov/pubmed/3810117

Cornejo, M., Mendoza, F. y Rojas, R. (2008). La investigación con relatos de vida: pistas y opciones del diseño metodológico. Revista Psyke,(17), 29-39.doi: 10.4067/S0718-22282008000100004

Ferrarotti, F. (2011). Las historias de vida como método. Acta sociológica,(56), 95-119. Recuperado de: http://revistas.unam.mx/index.php/ ras/article/viewFile/29459/27408

Fox, M. (2010). Women and men Faculty in Academic Science Egineering: Social-organizational Indicators and Implication. American BehavioralScientist, (53), 997-1012.doi: 10.1177/0002764209356234

Gilbert, J. (2011). La construcción social del científico. Notas sobre Identidad intelectual y social de comunidades científicas en Chile. Estudios Sociales, (119), 169-206. Recuperado de: http:// www.sistemascomplejos.cl/wp-content/uploads/2012/04/La-construcción-social-del-cient\%C3\%ADfico-Jorge-Gibert-Galassi2011.pdf

Gonzálvez, H. (2010). Migración Colombiana, género y parentesco: la organización social de los cuidados. (Tesis doctoral). Universidad de Granada, Granada, España.

Guerra, D. y Skewes, JC. (1999). La historia de vida como contradiscurso: pliegues y repliegues de una mujer. Revista Proposiciones, (19), 1-9. Recuperado de: www.sitiosur.cl/publicaciones/Revista_ Proposiciones/PR-0029-3265.pdf

Hall, O. (1948). The Stages of Medical Career. American Journal of Sociology, (53), 327-336.doi: 10.1086/220199

Health, C. (1984). Review essay: EveretCherrington Hughes (1897-1983): a note on his approach and influence. Sociology of Health and Illnes, (6), 218-237.doi: 10.1111/1467-9566.ep10778393

Holt, BWG. (1970). Social aspects in the emergence of chemistry as an 
exact science: The British Chemical Profession. The British Journal of Sociology,(21), 181-199.

Hughes, E. (1937). Institutional Office and the Person. American Journal of Sociology,(43), 404-413.doi: 10.1086/217711

Hughes, E. (1945). Dilemmas and Contradictions of Status. American Journal of Sociology,(50), 353-359.doi: 10.1086/219652

Knorr Cetina, K. (2005).La fabricación del conocimiento. Un ensayo sobre el carácter constructivista y contextual de la ciencia.Buenos Aires, Argentina: Universidad Nacional de Quilmes Editorial.

Kornblit, A. (2004). Historias y relatos de vida: una herramienta clase en metodologías cualitativas. En AL. Kornblit (eds.),Metodologías cualitativas: modelos y procedimientos de análisis, (pp. 15-34). Buenos Aires, Argentina: Biblos.

Kreimer, P. (1999). De probetas, computadoras y ratones. La construcción de una mirada sociológica sobre la ciencia. Buenos Aires, Argentina: Universidad Nacional de Quilmes.

Kyvik, S. (1990). Motherhood and scientific productivity. Social Studies of Sciences,(20), 149-160.Doi: 10.1177/030631290020001005

Lavados, J. (2006). Los negocios universitarios en el mercado del conocimiento. Santiago: J. C. Saìez Editor-CPU.

Longo, ME. (2009). Género y trayectorias laborales. Un análisis del entramado permanente de exclusiones en el trabajo. Revista Trayectorias,(11), 118- 141. Recuperado de: http://www.redalyc.org/pdf/607/ Resumenes/Resumen_60712751008_1.pdf

Lopez, E. yFindling, L. (2012). Maternidades, paternidades, trabajo y salud ¿Transformaciones o retoques?. Buenos Aires, Argentina: Editorial Biblios.

Martínez, S. (2012). Ser o no ser: tensión entre familia, subjetividad femenina y trabajo académico en Chile. Un análisis desde la psicología feminista. Revista de Estudios de Género La Ventana, (35), 133-163. Recuperado de: http://www.scielo.org.mx/scielo.php?pid=S140594362012000100006\&script=sci_abstract

Mason, M. y Goulden, M. (2004). Marriage and baby blues: Redefining gender equity in the academy. Annals of the American of Political and Social Science,(596), 86-103.Doi: 10.1177/0002716204268744

Montilva, M. (2008). Postergación de la maternidad de mujeres profesionales jóvenes en dos metrópolis latinoamericanas. Revista Utopía y 
Praxis Latinoamericana, (41), 69-79. Recuperado de: http:// w w w. s c i e l o.org. ve / s c i e l o.p h p ? p i d = S 1315 52162008000200004\&script=sci_abstract

Mora, C. (septiembre de 2010). Género, ciencia y mercado laboral. Seminario Género y ciencia, Universidad Alberto Hurtado, Santiago, Chile.

Morris, P.J. (2010). Does membership of royal society affect careers? A trio in twentieth-century organic chemistry. Notes and records of the Royal Society of London, (64), 77-87.

Ortíz, L. y Hernandez, M.(2009). La condición de género en la formación de investigadoras en la Universidad de Guadalajara. En Género y Trabajo en las Universidades, (sin pp.).Guadalajara, España: Instituto Municipal de las Mujeres en Guadalajara y Gobierno Municipal de Guadalajara.

Ortiz, F. y Larrazabal, S. (2015). Problematizando la noción de género. Revista Contenido. Cultura y Ciencias Sociales, (6), 6-13. Recuperado de: http://www.revistacontenido.com/wp-content/uploads/2015/ 12/RC6-Larazabal-Sofia-y-Ortiz-Francisca-Editorial.pdf

Ortiz, F. y Gonzálvez, H. (2016). Cómo explicar la organización social de los cuidados en Chile: una aproximación al proceso de envejecer. En Vera, A. (Ed.) Malestar social y Desigualdad en Chile(pp. 173-194). Santiago, Chile: Ediciones Universidad Alberto Hurtado.

O’Laughlin, E. y Bischoff, L. (2005). Balancing Parenthood and Academia: Work/Family Stress as influenced by gender and tenure status. Journal of Family,(26), 79-106.doi: 10.1177/0192513X04265942

Palomar, C. (2009). Maternidad y mundo académico. Revista Alteridades,(19), 55-73. Recuperado de: https://es.scribd.com/ document/251936904/Materniad-y-Mundo-Academico

Pereira, T. (2007). Afectos e intimidades. Santiago, Chile: Ediciones Universidad Católica.

PNUD. (2010). Género: los desafíos de la igualdad. Santiago, Chile: Informe de Desarrollo Humano en Chile.

Pujadas, J. (2002). El método biográfico: el uso de las historias de vida en ciencias sociales. Madrid, España: Centro de Investigaciones Sociológicas.

Ramos, C. (2012). Estructuras de comunicación en el campo de la ciencia social en Chile: un Análisis de redes. Revista REDES,(23), 7- 42.doi: 10.5565/rev/redes.438 
Rodigou, M., Burijovich, J., Domínguez, A. y Blanes, P. (noviembre en 2010). Trayectorias académicas: las marcas de género en la Universidad Nacional de Córdoba. Congreso Internacional Las políticas de equidad de género en prospectiva: nuevos escenarios, actores y articulaciones, Buenos Aires, Argentina.

Sautu, R. (2004). Estilos y prácticas de la investigación biográfica. EnSautu, R. (Comp.)El método biográfico(pp. 21-56).Argentina: Ediciones Lumiere.

Selva, C., Sahagún, MA.yPallarès, S. (2011). Estudios sobre Trayectorias Profesional y acceso de la mujer a cargos directivos: un análisis bibliométrico. Revista de Psicología del Trabajo y de las Organizaciones, (27), 227-242. Recuperado de: http://www.redalyc.org/pdf/ 2313/231322132007.pdf

Sharim, D. (2005). La identidad de género en tiempos de cambio: una aproximación desde los relatos de vida. Revista Psyke, (14), 19-32.doi: 10.4067/S0718-22282005000200002

Simbürger, E. y Undurraga, R. (2013). Jerarquías epistemológicas: Formas de invisibilizar el género en las carreras de sociología en Chile. En C. Mora, (Eds.) Desigualdad en Chile: la continua relevancia del género(pp. 171-195). Santiago, Chile: Ediciones Universidad Alberto Hurtado.

Smart, J.C. y McLaughlin, GW. (1985). Administrative service and research performance: a study of chemistr department heads. Research in higher education,(22), 31-42.doi: 10.1007/BF00992396

Stake, R.(2013). Estudios de casoscualitativos. En N. Denzin y Y. Lincoln (Coord.)Las estrategias de investigación cualitativa, 154-197. Madrid; España: Gedisa editorial.

Stefoni, C. (2010). Acceso y participación en la comunidad científica. Seminario Género y ciencia, Universidad Alberto Hurtado, Santiago, Chile.

Solé, C. y Parella, S. (2004). “Nuevas” expresiones de la maternidad. Las madres con carreras profesionales “exitosas”. Revista Española de Sociología,(4), 67-92. Recuperado de: https:/dialnet.unirioja.es/ servlet/autor?codigo $=44594$

Sonnert, G. y Holton, G. (1996). Career Patterns of Women and Men in the Sciences. American Scientist, (84), 63-71.Recuperado de: https:// www.researchgate.net/publication/234364700_Career_Patterns_ of_Women_and_Men_in_the_Sciences

Taylor, S. y Bogdan, R. (2000). Introducción a los métodos cualitativos de investigación. Barcelona, España: Paidós. 
Valdés, X. (2007). Vida en común. Familia y vida privada en Chile.Santiago, Chile: Lom Editores.

Valdés, X. (octubre de 2009). Metamorfosis de la familia y la vida privada. Cambios y tendencias en Chile. En la Universidad del BíoBío, BíoBio, Chile. 